\section{TRACK CALCULUS NOT CALORIES}

Chances are that many of your patients started the New Year with good intentions to lose a few pounds. Well, rather than tracking calories, why not encourage them to track their oral hygiene? Monitoring their actual activity versus what they believe they're doing might be the wake-up call they need to change

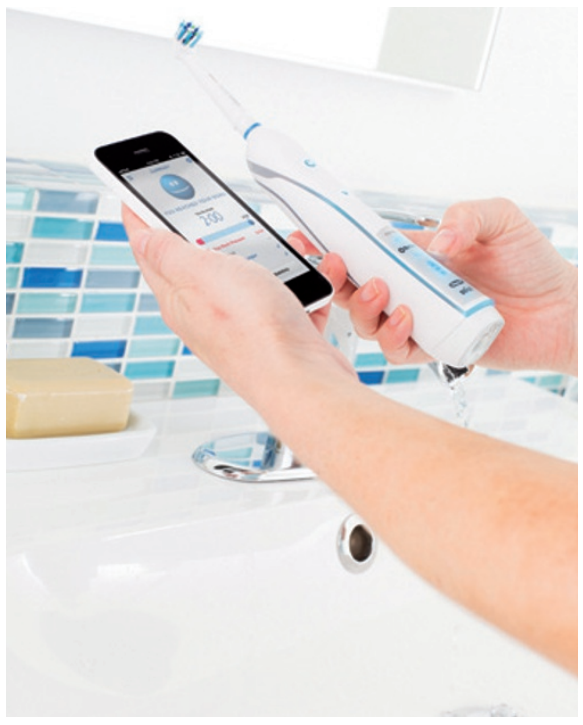

their oral hygiene habits for good.

Oral-B's new SmartSeries electric toothbrush can help patients to take ownership of their own oral health. This ingenious device allows dental professionals to programme patients' brushing routines onto their mobile to ensure they follow professional guidance between appointments. Control is firmly passed to the patient, but under the guidance of dental professionals. Brushing duration, mode and problem zones can all be highlighted and the information easily retrieved with the patient's consent. It's almost like having 'Big Brother' in the bathroom!

Whilst it is not possible to reverse the past and although surgical intervention cannot be ruled out in the future, if patients can learn to take care of their oral health, more serious intervention can be put off indefinitely. Oral-B's SmartSeries electric toothbrush can provide patients with a heightened level of motivation to look after their oral health, which has to be good news for everyone.

\title{
TAKE ACTION AGAINST PERIODONTITIS
}

New from oral healthcare specialist Curaprox is the perfect pocket minimiser for the treatment of chronic and aggressive periodontitis.

Adjusan combines the active ingredient doxycycline with a novel design for easy subgingival application, high tolerability and optimum efficiency.

The effective and uncomplicated single administration is easily applied via the practical cylinder cartridge. The local antibiotic has the ability to kill key microorganisms whilst remaining safe for the patient, as systemic exposure to doxycycline is minimal.

The most impressive characteristic of Adjusan is its innovative gel-like consistency. The initial flowable form allows you to reach the deep areas of the periodontal pocket, remaining in place as its viscosity increases in the aqueous environment. The active ingredient is released continuously at a sufficiently high concentration over at least 12 days, enabling pocket depth to be reduced and the attachment level to be enhanced.
Adjusan is also biodegradable to glycolic acid and lactic acid so there is no need for removal following treatment.

Take action against periodontitis gently but resolutely - contact the knowledgeable team at Curaprox today to find out more. For more information call 01480862084 or visit www. curaprox.co.uk.

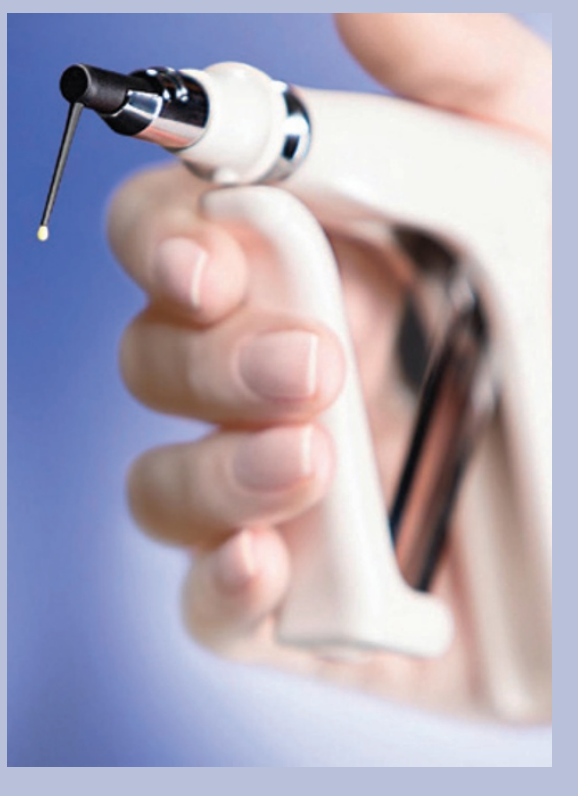

